\title{
Pulmonary hypertension: When the acute event leads to diagnosis. Case report in a patient with Ehlers-Danlos syndrome
}

\author{
Gianmarco Secco, Anna Giulia Falchi, Chiara Blatti, Bianca Giacomuzzi Moore, Anna Sabena, \\ Stefano Perlini, Francesco Salinaro
}

Emergency Department, IRCCS Policlinico San Matteo Foundation, University of Pavia, Italy

\begin{abstract}
Chronic thromboembolic pulmonary hypertension is a pulmonary vascular disease caused by the chronic obstruction of the major pulmonary arteries, usually being the consequence of recurrent episodes of pulmonary embolism. These events are usually unrecognized, and the consequent diagnostic delay leads to a deterioration of the clinical picture, thereby worsening the overall prognosis. Here we report the case of a 55-year-old man who was admitted to the Emergency Room (ER) for an acute exacerbation of chronic dyspnea, that he has been experiencing for several years, in the absence of any explanatory diagnosis. Acute pulmonary embolism was diagnosed in the setting of the ER, with bedside echography resulting crucial to the subsequent work up. A multidisciplinary approach led to proper treatment, follow-up management and positive subsequent outcome.
\end{abstract}

\section{Introduction}

Chronic thromboembolic pulmonary hypertension (CTEPH) is one type of pulmonary hypertension $(\mathrm{PH})$, caused by vascular obstruction and remodeling of the pulmonary arteries. CTEPH's cumulative incidence in the two years following a symptomatic acute pulmonary embolism episode has been reported to be 0.1 $9.1 \%{ }^{1}$ The wide margin of error is probably explained by the dif-

Correspondence: Gianmarco Secco, Emergency Department, IRCCS Policlinico San Matteo Foundation, University of Pavia, viale Golgi 19, 27100 Pavia, Italy.

E-mail: secco.gianmarco@gmail.com

Key words: Pulmonary hypertension; pulmonary embolism; echocardiography; Ehlers-Danlos syndrome; endarterectomy.

Conflict of interest: No one. This work was not supported by any grant.

Ethics approval and consent to participate: The manuscript does not contain any elements that would allow the recognition of the patient.

Received for publication: 27 May 2019.

Revision received: 15 January 2020.

Accepted for publication: 17 January 2020.

This work is licensed under a Creative Commons Attribution 4.0 License (by-nc 4.0).

(C) Copyright: the Author(s), 2020

Licensee PAGEPress, Italy

Emergency Care Journal 2020; 16:8310

doi:10.4081/ecj.2020.8310 ficulty to distinguish the analogous symptoms and first instrumental data of acute pulmonary embolism from clinical features of a pre-existent CTEPH. Approximately $30 \%$ of cases of chronic thromboembolism present with recurrent or overt episodes of acute events. We report the multidisciplinary approach and management of a CTEPH clinical case, that was initially misdiagnosed, and subsequently identified due to a further acute exacerbation. A brief analysis of the diagnostic work up with a revision of the related literature is also reported.

\section{Case Report}

A 55-year-old man came to the Emergency Room (ER) at the end of May 2018 because worsening of chronic dyspnea and new onset palpitations. His vital parameters were respectively: arterial blood pressure 135/95 $\mathrm{mmHg}$, heart rate $107 \mathrm{bpm}$, and 93\% $\mathrm{SatO}_{2}$ in room air. Clinical examination showed: valid heart sounds, with a 3/6 mesosystolic heart murmur in mesocardium, normosphygmic peripheral pulses, pitting edema of the legs, normal bilateral vescicular murmur with fine crackles at the lung bases. An arterial blood gas analysis was performed, showing $\mathrm{pO}_{2} 61 \mathrm{mmHg}, \mathrm{pCO}_{2} 27$ $\mathrm{mmHg}$, and lactate values in the normal range. The electrocardiogram showed a slightly tachycardic sinus rhythm, with signs of right ventricular overload. His clinical history reported: primary systemic hypertension treated with ramipril, previous tabagism, glaucoma, Ehlers-Danlos syndrome (EDS) (diagnosed in 1980) and a history of dyspnea [World Health Organization (WHO) grade II], starting from 2011. Upon further investigation the patient admitted worsening dyspnea in November 2017, at a time when a cardiac ultrasound scan revealed an estimated systolic pulmonary artery pressure (PAPs) of $53 \mathrm{mmHg}$, while a stress test had to be interrupted because of dyspnea. On May 1st, 2018, due to further dyspnea worsening (WHO II-IV), another cardiologic evaluation was carried out, with echocardiography revealing an estimated PAPs of 100 $\mathrm{mmHg}$, with a $4.5 \mathrm{~m} / \mathrm{s}$ peak tricuspid regurgitation velocity. The right ventricle (RV) was severely dilated and hypokinetic with a depressed systolic function. Also, the left ventricular systolic and diastolic function were found to be compromised. High priority hospitalization was therefore planned, due to pulmonary hypertension. Before admission, the patient entered the ER for further dyspnea worsening. Pulmonary embolism was suspected, and a transthoracic echocardiography (TTE) was performed. The latter showed a large endoluminal mobile mass (Figure 1) extending from the right atrium through the tricuspid valve into the right ventricular cavity, with concomitant dilation and systo-diastolic dysfunction of both the right and the left ventricles. Inferior vena cava diameter was $26 \mathrm{~mm}$, with decreased inspiratory collapse $(<50 \%$ with a sniff). An angio-computed tomographic (CT) scan (Figure 2) confirmed the presence of a bilateral massive pulmonary embolism, involving the main right and left branches, with a further thrombotic mass on the pulmonary artery bifurcation, and a filling defect of 
the right sovrahepatic vein. Due to the clinical severity, the evident systo-diastolic left ventricular dysfunction, as well as the presence of severe pulmonary hypertension concomitant to a right heart thrombus, a cardiac surgical approach was chosen in agreement with heart surgeons and cardiologists. Emergency pulmonary embolectomy was performed, finding a fresh bloody thrombus. Moreover, a chronic whitish organized thrombus extending from the distal right and left pulmonary artery to the peripheral branches

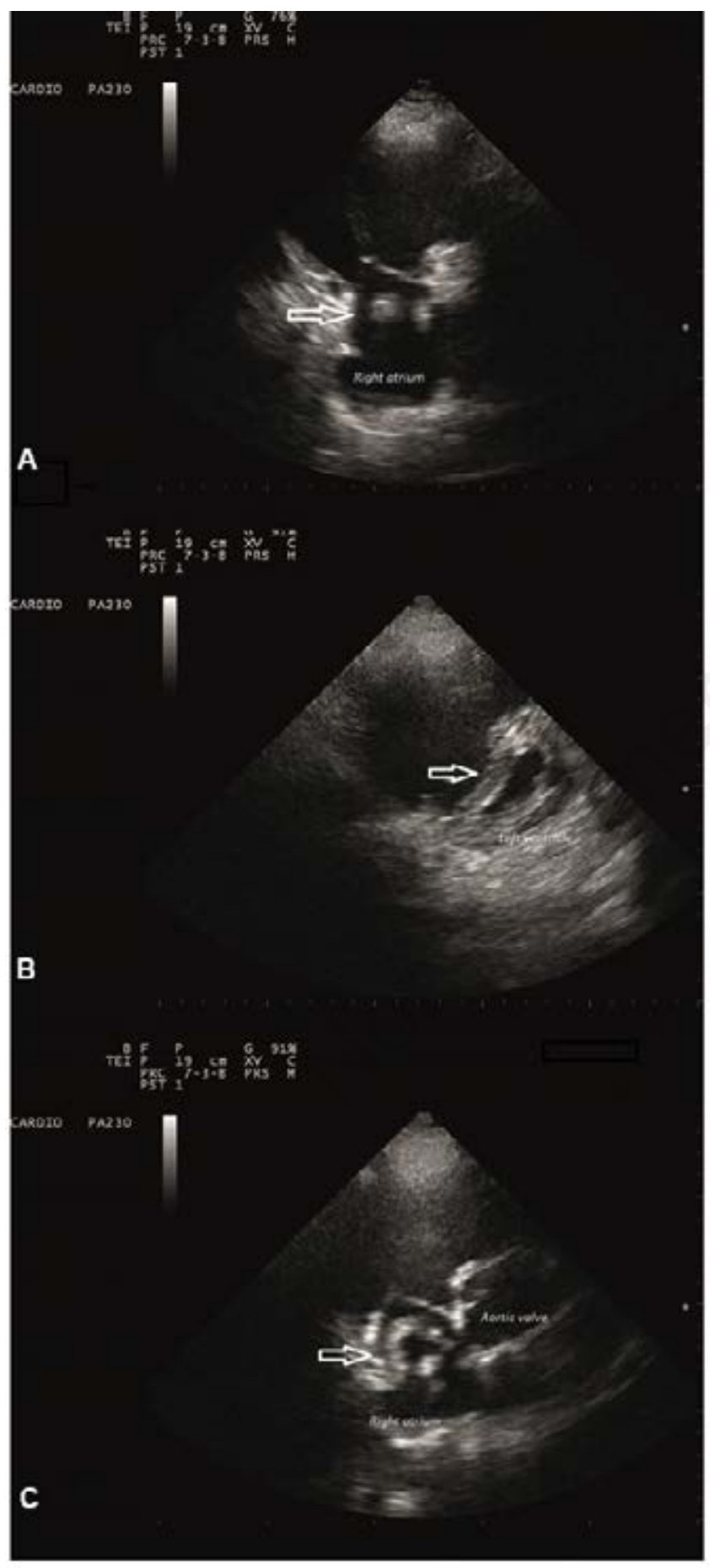

Figure 1. A) Four chambers view; $B$ and C) parasternal short axis view, at end-diastole demonstrating interventricular septal flattening, right ventricular dilatation and a large thrombus. was also found. To remove such a thrombus, endarterectomy was performed. During post-operative follow-up, right heart catheterization showed a pulmonary artery pressure of $22 \mathrm{mmHg}$ and a wedge pressure of $16 \mathrm{mmHg}$ confirming the final diagnosis. Doppler ultrasound of the lower limbs was also carried out, showing thrombosis of the left popliteal vein. Moreover, further investigations revealed positivity to anti ds-DNA antibodies. A vena cava filter was therefore positioned, given the high-risk patient profile, related to the probability of thrombophylic diathesis and the results of the previous angio-CT scan. The patient was then transferred to a rehabilitation facility for recovery, with oral anticoagulation therapy (warfarin), spironolactone $50 \mathrm{mg}$ and $\mathrm{N}$-acetylcystein $600 \mathrm{mg}$. An intensive follow-up was programmed after discharge. Five

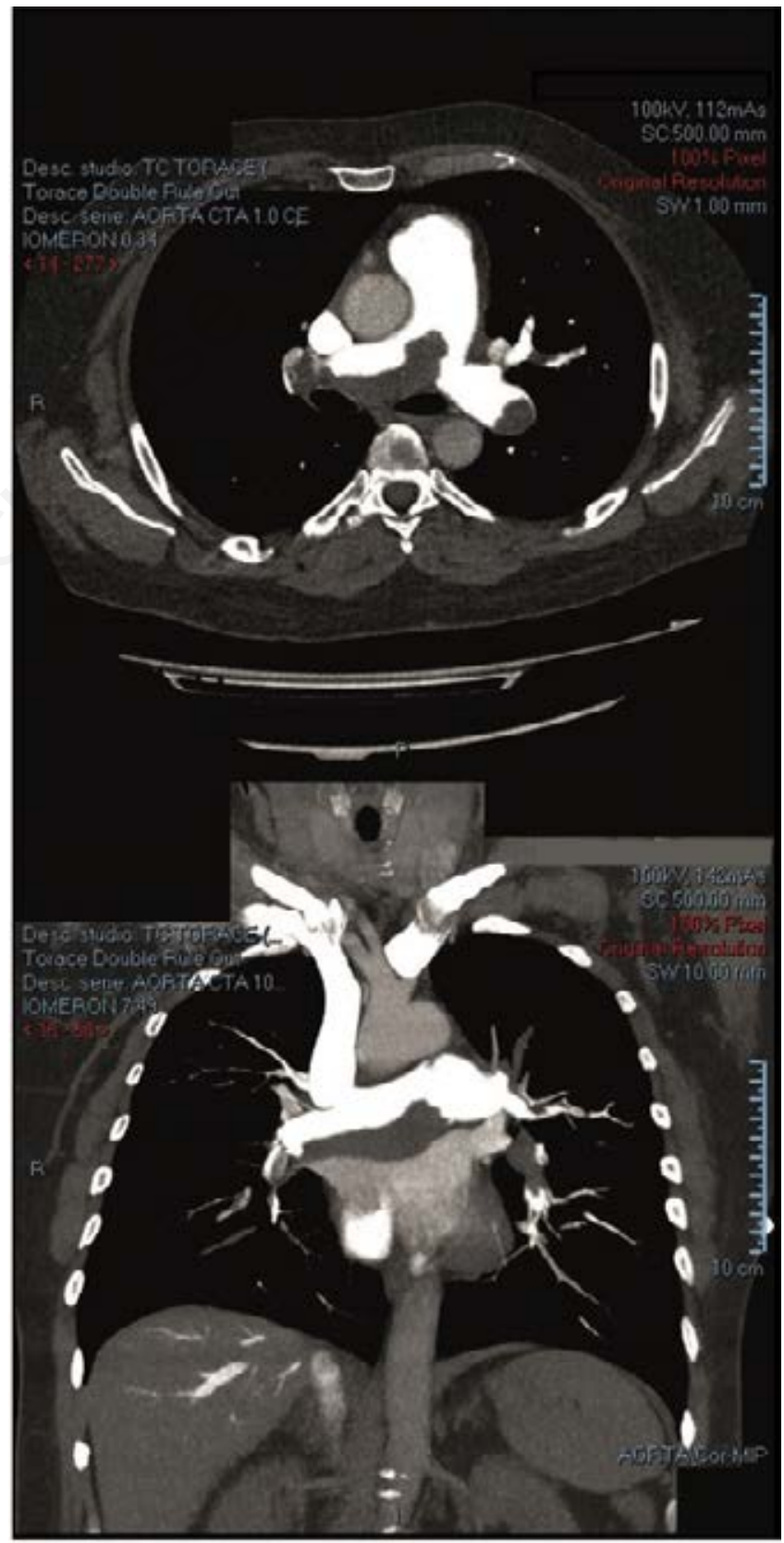

Figure 2. Angio-computed tomographic scan show a thrombotic mass located on the pulmonary arteries bifurcation. 
months later a second right heart catheterization confirmed the excellent surgery outcome, with pulmonary artery pressure of 13 $\mathrm{mmHg}$ and a wedge pressure $9 \mathrm{mmHg}$,

\section{Discussion}

The European Society of Cardiology assigns CTEPH to the IV group of pulmonary hypertension, being defined as a precapillary pulmonary hypertension. Its diagnosis is given when the pulmonary artery pressure is higher than $25 \mathrm{mmHg}$, and wedge pressure lower than $15 \mathrm{mmHg}$ upon right heart catheterization, in the presence of a thrombus or embolus either chronic or organized limiting the pulmonary artery flow, found after 3 months of anticoagulation therapy. ${ }^{1}$ Despite the notion that previous episodes of massive or recurring pulmonary embolism are the leading cause of CTEPH, evidence to prove such antecedents lacks in one fourth of the patients undergoing surgery for it. ${ }^{2}$ Approximately $30 \%$ of cases of chronic thromboembolism present with recurrent or overt episodes of acute events. ${ }^{3}$ The exact incidence of this disease is difficult to estimate, since the clinical presentation of CTEPH can mimic an acute embolic event, thus explaining the brief period of time elapsing between the recognition of an acute thromboembolic event and the development of CTEPH in some patients. ${ }^{4}$ Diagnostic delay is partly explained by the often non-specific presentation of the disease process and, early in its natural history, the subtlety of what could be symptomatic evidence on physical examination. The lack of prior history of acute thromboembolism is therefore a frequent obstacle in diagnosis. Usually, a long period with no symptoms elapses between the acute embolic event and first signs and symptoms of the chronic phase; such a frame of time is usually referred to as the honeymoon and can last for years, therefore making the causal connection between the two events rather difficult to disentangle. ${ }^{5}$ The symptoms of $\mathrm{PH}$ are non-specific and mainly related to progressive RV dysfunction. They include shortness of breath, fatigue, weakness, angina and syncope. The progressive dyspnea and exercise intolerance in these patients are often erroneously attributed to asthma, physical deconditioning, advancing age, interstitial lung disease, coronary artery disease, or psychogenic dyspnea. ${ }^{6}$ In our case, the acute worsening of symptoms and the high risk of hemodynamic failure, did not allow to perform right heart catheterization, to carefully measure pulmonary artery pressure and wedge pressure, to confirm CTEPH diagnosis. Anyway, the clinical history and the echocardiographic features suggested a high risk of chronic thromboembolism, as reported in guidelines. ${ }^{1}$ The acute embolic event and the elevated pulmonary pressure measured after surgical management confirmed final diagnosis of acute on CTEPH. By an epidemiologic standpoint in the Spanish registry of pulmonary embolism, the prevalence and incidence resulted to be respectively of 3.2 cases per million and 0.9 cases per million per year. ${ }^{7}$ Pathophysiologic explanations behind CTEPH range from the acute clotting event, to the lack of resolution of thromboembolism, to an imbalance between the two events, to a defect in the anticoagulation or fibrinolytic processes. In this cascade of events, direct consequences of CTEPH are the chronically increasing RV afterload and the augmenting wall stress; leading to an increase in overall pressure. The consequent RV hypertrophy, with wall thickening and the hypertrophy of the myocardial cells, eventually leads to RV failure, which is the main cause of death in CTEPH..${ }^{8,9}$ In the here reported clinical case a major element of the diagnostic work up and therapeutic planning has been the diagnosis of a thrombus in the right atrium, that was made possible thanks to bed-side echocardiography. Right heart thrombi (RHT) are a rare occurrence, noted in only $4 \%$ of all pulmonary embolism (PE) according to the International Cooperative Pulmonary Embolism Registry (ICOPER). ${ }^{10}$ Such an event may occur as the result of a clot travelling from distant deep veins to the right side of the heart. There is also the possibility that it may form in situ as a result of stagnation of blood in the heart, due to impaired or incomplete emptying of blood in the right heart, as seen in atrial fibrillation and some cardiomyopathies. The role of echocardiography in the diagnostic and prognostic evaluation of RHT is well established and continues to be an important tool, as it is easily available and bedside. TTE is usually sufficient for the diagnosis of right heart thrombi-in-transit and is considered a screening test, with 50 to $60 \%$ sensitivity for detection of right heart thrombi. It can anyhow underestimate the clot's size, whereas transesophageal echocardiography not only detects cardiac thrombus with higher accuracy, but it also allows diagnosis of pulmonary embolism with $80 \%$ sensitivity and $100 \%$ specificity in patients with suspected massive pulmonary emboli. ${ }^{11,12}$ Based on an observational study by the European Working Group on Echocardiography in 1989, right heart thrombi were grouped into three main morphological types. ${ }^{13}$ Type A thrombi are usually found in association with deep vein thrombosis and PE, thus thought to derive from large veins, transiting through the venous circulation before arriving to the right heart; they are highly mobile clots, elongated and serpiginous. Type B are believed to be developed in situ and to be associated to underlying cardiac anomalies. Type $\mathrm{C}$ are rather rare and morphologically resemble cardiac myxomas. Different treatment options are available, including IV anticoagulation therapy, systemic thrombolysis and surgical embolectomy. The choice of therapy is based on the physician's choice and clinical judgment, and is usually directed towards urgent surgical treatment or thrombolysis. Several case reports describe good short-term outcomes for surgically as well as medically managed patients. In our case the high suspicion of acute on chronic pulmonary thromboembolism, due to echo features and past history, led to choose surgical treatment, i.e. pulmonary embolectomy. When acute pulmonary thromboembolism is accompanied by chronic pulmonary embolism (acute on chronic thromboembolism), pulmonary endarterectomy should be considered in addition to pulmonary embolectomy. However, in our patient, was performed a pulmonary embolectomy, and also endarterectomy because a chronic organized thrombus was found. with a successful recovery. A similar case report with surgical management has been described in other paper. ${ }^{14}$ Another interesting element of this case report concerns the fact that the patient suffered from EDS. EDS comprises a group of hereditary connective tissue disorders in which collagen synthesis and fibrogenesis is impaired. This heterogeneous disorder has an estimated prevalence of 1:5000 to 1:25,000 new births. ${ }^{15}$ This kind of patients have a bleeding tendency manifested by ecchymoses, hematomas, and the rupture of blood vessels. However, some rare thrombotic events are described in literature associated with the presence of positive antiphospholipid antibodies, although PE in a patient with EDS is really rare. ${ }^{15}$ Furthermore, EDS has been associated with transient antiphospholipid antibodies elevations. ${ }^{16}$ As far as our patient is concerned, an extensive work-up for hypercoagulable disorders was performed, resulting to be negative for the main known coagulation factors mutations. It has to be took into account that EDS implies that collagen that supports blood vessels in affected patients results to be unusually weak and elastic, making blood vessels more prone to injury. Subsequently, exposition of extracellular matrix, given by the damage to the endothelial cells, 
leads to the engagement of the hemostatic system including platelet adhesion and activation. ${ }^{17}$ The thrombotic events in our patient occurred at a later stage of his life; and could be attributed to other factors, despite him not having any risk factors prior to the time of thrombosis. A thrombotic event could thus be associated to possible endothelial damage previously described as being part of EDS spectrum of characteristics.

\section{Conclusions}

Chronic thromboembolic pulmonary hypertension is a rare complication of acute PE, but it can also occur in patients without a history of acute PE or in whom deep vein thrombosis is underdiagnosed. CTEPH remains a disease frequently misdiagnosed. This clinical case shows how the acute event, even if rarely found in literature, can be diagnosed in an Emergency setting thanks to bedside echography and angio-CT scan imaging; allowing a correct etiologic diagnosis of a disease that has most probably affected the patient for a long period of time. The multidisciplinary approach of such a case highlights how proper dialogue amongst specialties can lead to an accurate therapeutic approach, shortening work up timings and in the end leading to a better outcome.

\section{References}

1. Galiè N, Humbert M, Vachiery JL, et al. [2015 ESC/ERS Guidelines for the diagnosis and treatment of pulmonary hypertension]. Kardiol Pol 2015;73:1127-206. [Article in Polish].

2. Pepke-Zaba J, Delcroix M, Lang I, et al. Chronic thromboembolic pulmonary hypertension (CTEPH): results from an international prospective registry. Circulation 2011;124:1973-81.

3. Lang IM. Chronic thromboembolic pulmonary hypertension: not so rare after all. N Engl J Med 2004;350:2236-8.

4. Guérin L, Couturaud F, Parent F, et al. Prevalence of chronic thromboembolic pulmonary hypertension after acute pulmonary embolism. Prevalence of CTEPH after pulmonary embolism. Thromb Haemost 2014;112:598-605.
5. D'Armini AM, Zanotti G, Pozzi M, et al. [Surgical treatment of chronic thromboembolic pulmonary hypertension with pulmonary endarterectomy]. G Ital Cardiol (Rome) 2006;7:45463. [Article in Italian].

6. Pengo V, Lensing AW, Prins MH, et al. Incidence of chronic thromboembolic pulmonary hypertension after pulmonary embolism. N Engl J Med 2004;350:2257-64.

7. Escribano-Subias P, Blanco I, Lopez-Meseguer M, et al. Survival in pulmonary hypertension in Spain: insights from the Spanish registry. Eur Respir J 2012;40:596-603

8. Delcroix M, Vonk Noordegraaf A, Fadel E, et al. Vascular and right ventricular remodelling in chronic thromboembolic pulmonary hypertension. Eur Respir J 2013;41:224-32.

9. Van de Veerdonk MC, Bogaard HJ, Voelkel NF. The right ventricle and pulmonary hypertension. Heart Fail Rev 2016;21:259-71.

10. Torbicki A, Galie N, Covezzoli A, et al. Right heart thrombi in pulmonary embolism: results from the International Cooperative Pulmonary Embolism Registry. J Am Coll Cardiol 2003;41:2245e2251.

11. Schwartzbard AZ, Tunick PA, Rosenzweig BP, Kronzon I. The role of transesophageal echocardiography in the diagnosis and treatment of right atrial thrombi. J Am Soc Echocardiogr 1999;12:64-9.

12. Pruszczyk P, Torbicki A, Pacho R, et al. Noninvasive diagnosis of suspected severe pulmonary embolism: TTE versus spiral CT. Chest 1997;112:722-8.

13. Finlayson GN. Right heart thrombi: consider the cause. Can J Cardiol 2008;24:888

14. Lee H. Life-threatening acute on chronic pulmonary thromboembolism requiring extracorporeal membrane oxygenation. Korean J Thorac Cardiovasc Surg 2018;51:205-8.

15. Royce PM, Steinmann B, eds. Connective tissue and its heritable disorders: molecular, genetic, and medical aspects. 5th edition. New York: Wiley-Liss; 2002. pp 431-523.

16. Asherson RA, Bosman C, Tikly M, et al. Ehlers-Danlos syndrome type IV in a young man. J Rheumatol 2006;33:2091-6.

17. Jiménez-Encarnación E, Vilá LM. Recurrent venous thrombosis in Ehlers-Danlos syndrome type III: an atypical manifestation. BMJ Case Rep 2013;2013: pii: bcr2013008922. 